environmental factors and genetic predisposition. Auditory hallucinations $(\mathrm{AH})$ are a specific and disruptive symptom of schizophrenia affecting around $70 \%$ of patients [1].

In order to identify gene expression biomarkers of auditory hallucinations in schizophrenia, we have conducted a networkbased transcriptomic analysis of 45 individuals, 15 with $\mathrm{AH}$ (SA), 15 without AH (SNA) and 15 matched controls (CNT).

Gene expression data from blood samples were generated using Affymetrix Human Gene 2.0 ST Genechips. The RMA normalization method was applied for gene summarization and the most variably expressed probesets $(4,508)$ between subject (or experimental) groups were selected. These dataset was analyzed using the weighted gene co-expression network analysis (WGCNA) package in R.

The gene co-expression network analyses identified 11 different gene modules based on their topological overlap. These modules were related to phenotypic information allowing us to identify modules differentially expressed according with specific phenotypic traits.

To identify potential blood biomarkers of schizophrenia and auditory hallucinations, we selected modules including genes which expression differed significantly mainly in the three subject groups (CNT, SNA and SA), but showing no changes due to the other analyzed traits such as age or medication.

Next, we chose genes from the selected modules with a high statistically significance $(\mathrm{p}<0.01)$. A total of 15 genes were found and differences in their expression in CNT, SNA and SA samples were tested by RT-qPCR. We confirmed differences in expression of two genes. The expression of the Eukaryotic Translation Initiation Factor 2D gene (EIF2D) was increased in schizophrenic patients versus controls $\mathrm{p}<0.001$. The expression of the Thymocyte Selection-Associated High Mobility Group Box gene (TOX) was reduced in the schizophrenic group $\mathrm{p}<0.01$.

To determine whether the 15 evaluated genes or a subset of them might be useful as biomarkers for schizophrenia, we interrogated the expression of each of them (as fold change compared to control average) using binary logistic regression. We obtained a predictive equation in which the EIF2D and TOX gene expression could differentiate schizophrenia patients versus controls: $-0.106+$ $[2.957 \times$ EIF2D $]+[-4.198 \times$ TOX $]$. Applying this equation to our sample, the schizophrenic patients could be separated from healthy controls with $86.5 \%$ accuracy.

Then, we applied the same analysis to identify potential biomarkers of AH. In this case, the expression of RNPS1, NRD1, CPQ and APOBEC3G were able to differentiate between SA and SNA with an $80.8 \%$ accuracy in our sample: $0.29+[2.596 \times$ RNPS1 $]+[-4.295 \times \mathrm{NRD} 1]+[2.821 \times \mathrm{CPQ}]+[-2.586 \times$ APOBEC 3G].

Using a network-based transcriptomic analysis, we have identified a set of genes that might be useful as blood biomarkers of schizophrenia and auditory hallucinations. Moreover, these genes pointed out new pathways involved in this disease.

\section{P.1.a.008 5-HTTLPR shows association with younger age at suicide: preliminary results from the Hungarian suicide biobank}

J. Bokor ${ }^{1}$, X. Gonda ${ }^{2}$, P. Dome ${ }^{2}$, G. Faludi ${ }^{2}$, E. Dinya ${ }^{3}$, A. Laszik ${ }^{1} \quad{ }^{I}$ Semmelweis University, Department of Forensic and Insurance Medicine, Budapest, Hungary; ${ }^{2}$ Semmelweis University, Department of Psychiatry and Psychotherapy,
Budapest, Hungary; ${ }^{3}$ Semmelweis University, Institute of Digital Health Sciences, Budapest, Hungary

Background: Although the majority of suicides are committed by psychiatric patients, suicide is a multicausal phenomenon with a strong biopsychosocial background, including a significant contribution of genetic factors. As suicide still contributes to a large number of unnecessary, and possibly preventable deaths worldwide, constituting the leading cause of death among young males, we lack effective methods for predicting, screening, and preventing suicide. Thus our aim is to develop a complex model incorporating several levels of evidence from genetics through biochemistry as well as social and psychosocial determinants of suicidal behaviour. The 5-HTTLPR polymorphism of the serotonin transporter gene has previously been implicated in suicidal behaviour and violent completed suicides. The aim of our present study was to investigate the association between 5HTTLPR and suicidal behaviour in our currently developing suicide biobank.

Methods: During autopsy sample DNA samples were obtained for 5-HTTLPR genotyping from 200 subjects deceased due to suicide and 200 controls deceased due to other causes. Chi-square tests and logistic regression analyses were performed according to additive, dominant and recessive models to analyse the possible association between 5-HTTLPR genotype distribution and suicide.

Results: Ratio of violent and non-violent suicides was $81 \%$ and $19 \%$ in our suicidal subsample, respectively. No significant difference was found in the distribution of 5-HTTLPR genotypes between the suicidal and control samples according to any genetic models (additive: $\chi 2=0.602, \mathrm{p}=0.740$; dominant: $\chi 2=0.404$; $\mathrm{p}=0.525 ;$ recessive $\chi 2=0.457 ; \mathrm{p}=0.499)$. No difference was found between violent and nonviolent suicides with respect to genotype distribution within the suicidal sample (additive: $\chi 2=1.771, \mathrm{p}=0.412$; dominant: $\chi^{2}=1.555, \mathrm{p}=0.212$; recessive $\left.\chi^{2}=0.913 ; \mathrm{p}=0.339\right)$. Using logistic regression, a significant association was found between sl genotype and suicide at a younger age in the suicidal subsample according to the additive model $(\mathrm{OR}=1.037 ; \mathrm{p}<0.001 ; \mathrm{df}=1 ; \mathrm{B}=0.036)$.

Conclusions: Although our preliminary study found no evidence for an association between 5-HTTLPR genotype and suicide, a significant association was found between the sl genotype and younger age at suicide, indicating an important role of this genetic variant in the emergence of suicidal behaviour which may deepen our understanding of the dynamics of suicide risk and may help us identify targets for early screening and intervention. Several limitations must be mentioned possibly contributing to our negative results. Our control group included subjects deceased due to accidents where suicidal intent cannot be ruled out. Furthermore, due to a lack of suicidal autopsy we could not gain data concerning psychiatric illness in our suicidal sample and we could not gather information on distal and proximal life stressors which are known to play a role in the effect of 5-HTTLPR at least in depression. Given the difficulty of studying suicidal behaviour due to the relatively low number of cases, our results argue for the further development of our suicide biobank and the investigation of further novel genotypes associated with endophenotypes of suicidal behaviour in living subjects. 